\title{
Two Successful Successive Pregnancies in a Woman with CML Treated with Dasatinib and Temporary Peg-Interferon
}

Gérard Dine, Marc Levert, Yves Rehn, Nadia Ali Amar, Said Brahimi, Baptiste Gaillard, Isabelle Bocq and Gabriela Fumagalli

Department of Hematology, Hopital des Hauts Clos, Troyes 10000, France

\begin{abstract}
With TKIs, the management of CML has radically changed. Here we present the case of a woman diagnosed with CML during two pregnancies. Medullary and complete cytogenetic remission was achieved with Imatinib. However, she developed major muscular intolerance. This patient expressed the wish to have children. Hematology experts advised to delay her plan to become pregnant. According to BCR/ABL transcripts levels, they also decided to substitute Imatinib for Dasatinib. A major molecular response was subsequently observed with disappearance of muscular toxicity. It was then decided to interrupt Dasatinib treatment and to substitute with pegylated interferon for the duration of the pregnancy. After a normal full term pregnancy, she gave birth to a baby boy. Dasatinib treatment was reintroduced. A year later, the patient became pregnant for a second time. Dasatinib treatment was interrupted and substituted with pegylated interferon. After a normal pregnancy, she gave birth to a baby girl. Dasatinib treatment was then reintroduced. This case report highlights the possibility for CML patients whose disease is stabilized to interrupt Dasatinib treatment and substitute with pegylated interferon during pregnancy without jeopardizing the patient outcome or exposing the fetus to TKI associated teratogen risks.
\end{abstract}

Key words: CML, TKIs, pregnancy, Dasatinib, BCR-ABL.

\section{List of abbreviations}

TKI Tyrosine kinase inhibitor

CML Chronic myeloid leukemia

FISH Fluorescent in situ hybridization

HLA Human leukocyte antigen

$\mathrm{Hb} \quad$ Hemoglobin

\section{Introduction}

The management of chronic myeloid leukemia (CML) in its initial stage has been totally revolutionized by the emergence of TKIs (tyrosine kinase BCR/ABL inhibitors). Today, the latest developments of 1st, 2nd and 3rd TKI generations have allowed the management of all the different stages of CML. If left untreated, this myeloid hemopathy can potentially lead to acute transformed CML, which is

Corresponding author: Gérard Dine, M.D. Ph.D., professor, research field: hematopoiesis, hematological devices and iron disorders. E-mail: gerard.dine@ch-troyes.fr. known to have a poor prognosis. However, the late phase of CML progression is no longer considered fatal and lifelong treatment with TKIs enable it to be kept under control. The impressive results obtained from TKI clinical trials have radically changed the disease and patient management guidelines. Physicians are now facing new clinical challenges. The median age of patients at the time of CML diagnosis is approximately 60 years old. However, $10 \%$ of patients are of childbearing age and some patients could therefore wish to conceive and have children. The challenge faced by CML patients treated with TKIs during the pregnancy is the teratogen risk associated with TKIs for the fetus [1]. The treatment could potentially be interrupted for the duration of the pregnancy, but this may expose the patient to a relapse, which could subsequently have implications on the disease progression. Current international literature reports 
numerous planned and unplanned pregnancies for CML patients while being treated with 1 st or 2 nd generation TKIs [2-4]. For patients whose disease progression has been stabilized, a therapeutic alternative may be to substitute their TKI treatment with pegylated interferon during the pregnancy: This option would take into account both the mother's and the fetus' health as by continuing a CML treatment it would allow the mother to remain in CML remission (initially induced by the TKI) and it would avoid any exposure to the TKI-associated teratogen risks for the fetus. Several teams of experts in hematology have successfully used this strategy of therapy replacement/substitution [5,6]. Here we present the case of a patient for whom we used this therapeutic strategy: She was in Dasatinib-induced major molecular remission and her treatment was substituted twice with pegylated interferon to allow her to have two successive full-term pregnancies. The patients did not have any specific complications during her pregnancies and so far, the treatment substitution did not have any consequences for the two children. She currently remains in molecular and hematologic remission after reintroduction of Dasatinib treatment.

\section{Case Description}

This female patient was born in April 1978 and she worked as an accountant. She did not have any medical and surgical history. In September 2003, during the first month of a twin pregnancy, she was diagnosed with an aggressive form of CML with hyperleukocytosis and myelemia at $210,000 / \mathrm{mm}^{3}$, and hemoglobin level at $11 \mathrm{~g} / \mathrm{dL}$.

After diagnosis, the patient was treated with hydroxycarbamide (1,500 mg/daily) and allopurinol $(100 \mathrm{mg} /$ daily) and an elective abortion was performed. Renal and hepatic functions were normal but the Sokal score was high. The myelogram showed granular mass hyperplasia without leukemic hiatus. The osteo-medullary biopsy confirmed the diagnosis of myeloproliferative syndrome with peritrabecular myelofibrosis.

The medullary karyotype performed on 20 mitoses demonstrated the presence of the $t(9 ; 22)$ chromosome translocation. The FISH (Fluorescence in situ hybridization) analysis confirmed a genetic abnormality integrated within a complex translocation between chromosomes 9 and 22 and chromosomes 5 and 13. No deletion of the extra signal from the ABL gene probe could be detected. Despite this genetically-complex case, a diagnosis of CML associated with a conventional $\mathrm{t}(9 ; 22)$ chromosomal translocation was made.

The analysis of HLA (human leukocyte antigen) markers within the family did not identify any potential related donors. This patient was registered on the French register for bone marrow transplantation. The hematological situation could not be fully controlled under hydroxycarbamide treatment: a persistent hemoglobin level of $9.5 \mathrm{~g} / \mathrm{dL}$ was observed.

Treatment with Imatinib at $400 \mathrm{mg} /$ day was started in November 2003. Shortly after, the peripheral hematologic remission was observed. However, she developed an intolerance with arthralgias and intense muscular cramps of grade 3 according to Common Toxicity Criteria for Adverse Events (Ver.4.0) and 3 weeks after starting the Imatinib treatment, the dose was reduced to $200 \mathrm{mg} /$ day.

Clinical improvement (medullar remission) was observed with the Imatinib treatment at $200 \mathrm{mg} /$ day from January 2004. The Imatinib dosage was increased to $300 \mathrm{mg} /$ day in September 2004. The BCR/ABL transcript ratio was measured at $16 \%$ in October 2004 and despite the Imatinib increase to $300 \mathrm{mg} /$ day, medullary analyses remained stable.

After 10-months Imatinib treatment and despite a non-optimized dosage, a complete cytogenetic response was confirmed in September 2004 after analysis of 20 mitoses.

The Imatinib dosage was increased to $400 \mathrm{mg}$ /day in January 2005 . This was immediately followed by the occurrence of a grade three muscular toxicity with 
hypocalcemia, hypophosphoremia and hypomagnesemia: These deficiencies were not easily manageable even with oral supplements. In May 2005, a serious sun photosensibilisation was reported. The deficiencies in calcium, phosphorus and magnesium persisted. The Imatinib dosage was again reduced to $300 \mathrm{mg} /$ day followed by clinical improvement. The BCR/ABL transcript ratio was 3.40\% in October 2005.

The karyotype analysis performed in January 2006 confirmed total cytogenetic remission. Throughout the year 2006, the Imatinib dosage varied between 300 $\mathrm{mg} / \mathrm{day}$ and $400 \mathrm{mg} /$ day depending on the occurrence of muscular intolerances associated with reported calcium, phosphorus and magnesium deficiencies. The $\mathrm{BCR} / \mathrm{ABL}$ transcript ratio was unsatisfactory at $6.40 \%$ in December 2006. However, the karyotype analysis performed in January 2007 showed that total cytogenetic remission remained.

Throughout the year 2007, the tolerance to Imatinib treatment varied according to the administered dosage (300 mg/day or $400 \mathrm{mg} /$ day).

At the beginning of the summer in 2007, the patient wished to become pregnant and was thinking of stopping her Imatinib treatment on her own initiative. The team convinced her to delay her decision to become pregnant. Analysis of the BCR/ABL transcript ratio performed in August 2007 showed that it was $8.40 \%$. A molecular progression under treatment was observed although the treatment remained poorly tolerated.

The search for genetic mutations was negative. The Imatinib dosage was at $848 \mathrm{ng} / \mathrm{mL}$, which was below the $1,000 \mathrm{ng} / \mathrm{mL}$ threshold. In October 2007, a panel of hematologists suggested substituting Imatinib for Dasatinib at $100 \mathrm{mg}$ once daily. Evaluation of the BCR/ABL transcript ratio performed in November 2007 and December 2007 indicated a decrease to $2.20 \%$ and $1.01 \%$, respectively. During a visit in December 2007, a clear clinical improvement was observed with the Dasatinib treatment at $100 \mathrm{mg}$ once daily.
During the 1st semester of 2008, the adverse events of muscular intolerance disappeared and the levels of calcium, phosphorus and magnesium returned to normal. In April 2008, the BCR/ABL transcript ratio was measured at $0.99 \%$.

The major molecular response was observed in January 2009 with a BCR/ABL transcript ratio of $0.0099 \%$ and this was confirmed in March 2009 with a transcript ratio at $0.0085 \%$.

Following these results and the acceptable tolerance to treatment with Dasatinib, the patient reiterated her strong wish to become pregnant during the summer of 2009. The patient had a normal professional life. In October 2009, a panel of hematology experts advised on stopping Dasatinib and substituting the treatment for pegylated interferon at a dose of 45 $\mu \mathrm{g} /$ week (Fig. 1).

The biological diagnosis of pregnancy was confirmed in January 2010. The pegylated interferon treatment was well tolerated and no problems were reported for the first 3 months of pregnancy. Therefore, the interferon dosage was not increased to the 90 $\mu \mathrm{g} /$ week dose recommended in the prescribing information.

The first analysis of the BCR/ABL transcript performed during the pregnancy in April 2010 gave a ratio of $0.0610 \%$ (Fig. 1). The administered dosage of pegylated interferon was not increased. In July 2010 in the 7th month of pregnancy, the BCR/ABL transcript ratio was $0.0410 \%$ (Fig. 1).

At the end of a full term pregnancy, the patient gave birth under epidural analgesia to a $3.21 \mathrm{~kg}$ baby boy (apgar score of 10 after $5 \mathrm{~min}$ ) on the September 26, 2010.

During a visit in November 2010, her general health status was normal. The BCR/ABL transcript ratio was $0.0630 \%$ (Fig. 1). The treatment with pegylated interferon was stopped and Dasatinib at $100 \mathrm{mg}$ once daily was reintroduced.

Analyses of the BCR/ABL transcript ratio in December 2010 and February 2011 indicated values of 


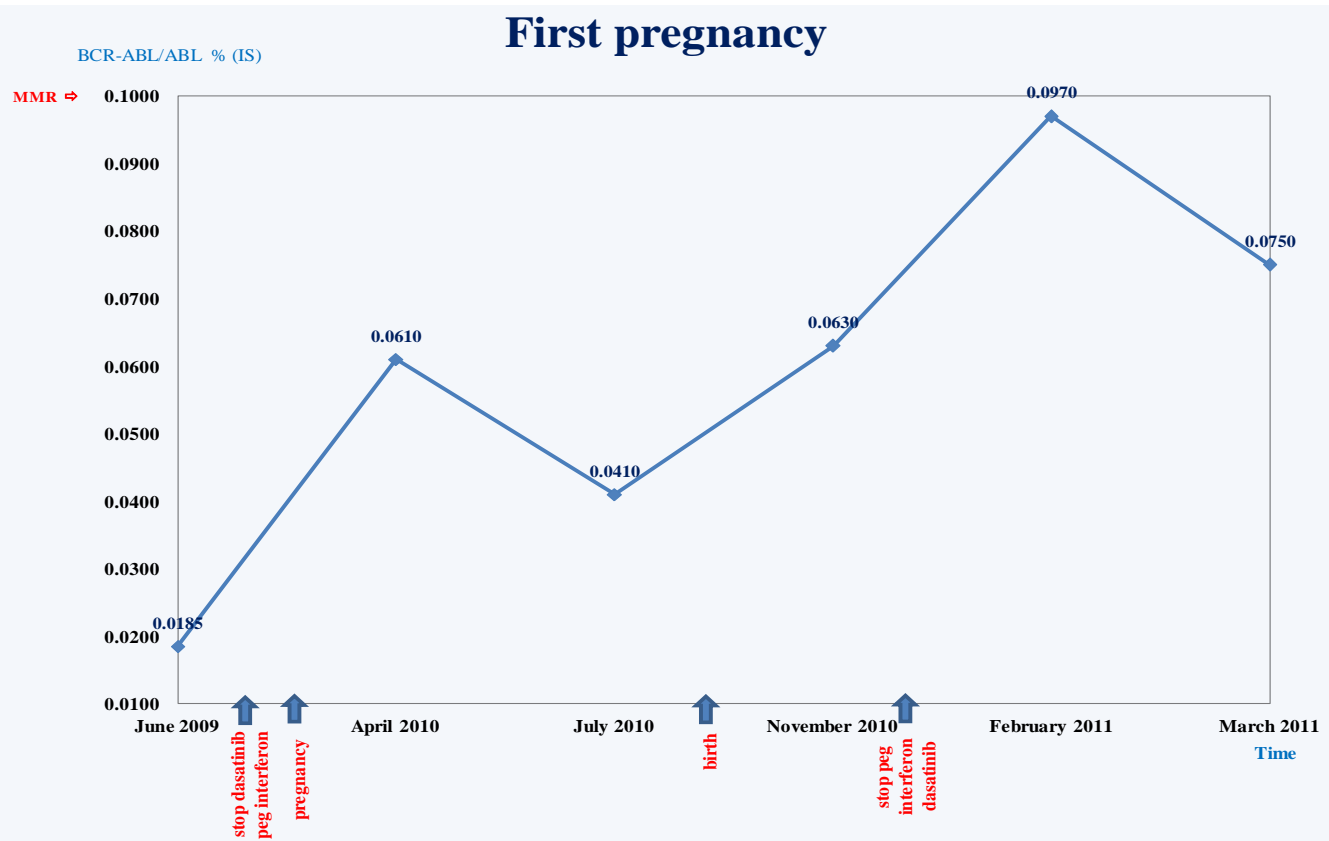

Fig. 1 BCR-ABL monitoring during the first pregnancy. The BCR-ABL/ABL transcript ratio according the international scale (IS) was monitored between June 2009 and March 2011, throughout the first pregnancy. The major molecular response (MMR) status is indicated at the BCR-ABL/ABL ratio of $0.1 \%$ and the occurrence of events such as treatment substitution, pregnancy confirmation and birth are also all indicated on the figure.

$0.0118 \%$ and $0.0970 \%$, respectively (Fig. 1). The psychomotor development of the child was totally acceptable and the patient presented a clinically normal situation. The hemogram was normal and the Dasatinib treatment was well tolerated. The patient started taking progesterone only oral contraception. In March 2011, the $\mathrm{BCR} / \mathrm{ABL}$ ratio was $0.0750 \%$ (Fig. 1).

At the end of April 2011, the patient had nausea and performed a pregnancy test, which was positive despite taking an oral contraceptive. The Dasatinib treatment was interrupted. An evaluation of the BCR/ABL transcript ratio performed in April 2011 gave a value of $0.0730 \%$ (Fig. 2). The pregnancy was confirmed at the end of May 2011 by echography and the patient wished to keep the baby.

Treatment with pegylated interferon at a dose of 45 $\mu \mathrm{g} /$ week was started. The BCR/ABL transcript ratio in June 2011 was $0.0890 \%$ (Fig.2). The pegylated interferon treatment was well tolerated and no problems were reported during the first 3 months of pregnancy.

The biomolecular surveillance done in August 2011 and October 2011 gave BCR/ABL transcript ratios of $0.0660 \%$ and $0.0741 \%$, respectively (Fig. 2). Gynecological routine examination indicated that both the patient's clinical situation and the echography were normal one month before the expected full term date.

The patient gave birth under epidural analgesia to a $3.16 \mathrm{~kg}$ baby girl (apgar score of 10 after $5 \mathrm{~min}$ ) 3 weeks before full term on the December 15, 2011.

Immediately in post-partum, the patient presented a mononucleosis syndrome contracted from a familial contamination (from her husband) and this caused a hepatic toxicity with a significant elevation in transaminase levels. The treatment with pegylated interferon was stopped and Dasatinib was not reintroduced. No major clinical consequences were observed. However the patient developed a neutropenia fluctuating between $1000 / \mathrm{mm}^{3}$ and $1,200 / \mathrm{mm}^{3}$. The transaminase levels returned to normal after 3 weeks. The Dasatinib at $100 \mathrm{mg} /$ day was reintroduced one month after giving birth. Evaluation of the BCR/ABL transcript ratio performed 3 days before the birth gave a value of $0.0610 \%$. After 


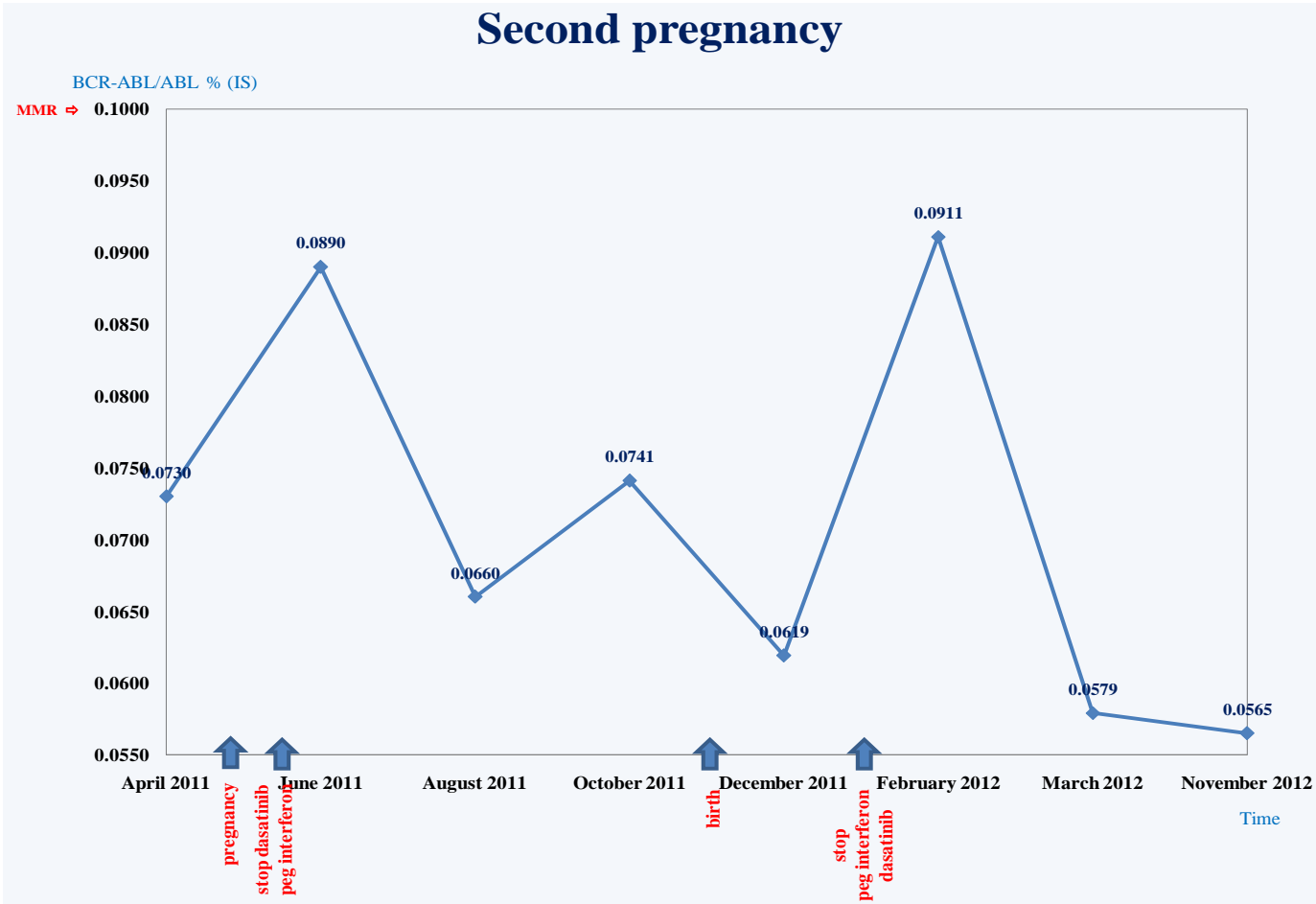

Fig. 2 BCR-ABL monitoring during the second pregnancy. The BCR-ABL/ABL transcript ratio according the international scale (IS) was monitored between April 2011 and November 2012, throughout the second pregnancy. The major molecular response (MMR) status is indicated at the BCR-ABL/ABL ratio of $0.1 \%$ and the occurrence of events such as treatment substitution, pregnancy confirmation and birth are also all indicated on the figure.

reintroduction of Dasatinib in January 2012, this ratio was $0.0936 \%$. Analyses performed in February 2012 and March 2012 (i.e., one and two months after Dasatinib reintroduction) gave values of $0.0911 \%$ and 0.0579\%, respectively (Fig. 2).

During a hematologic visit in May 2012, both the general health status and the clinical examination were normal. The Dasatinib treatment was well tolerated. A residual post-viral neutropenia of 1,500 polynuclear neutrophils $/ \mathrm{mm}^{3}$ persisted. The progesterone only oral contraception prescribed after the second pregnancy was replaced with an intra-uterine device for the patient's personal convenience and contraceptive security. In November 2012, the BCR/ABL transcript ratio was $0.0565 \%$ (Fig. 2). Currently, the patient has a fulfilling personal and family life: She is the mother of a 2.5-year old boy and 1.5-year old little girl. One and half years after the second pregnancy, she is still in major molecular remission under Dasatinib at 100 $\mathrm{mg} /$ day.

\section{Discussion}

This observation clearly contributes to the evidence that a CML patient can nowadays have a normal pregnancy despite the need for TKI therapy. After reviewing the international literature and from our experience, it is now possible to consider a pregnancy when under a long-term TKI treatment.

In our case report, the patient was initially treated with hydroxycarbamide. Because of lack of hematologic response, she started a TKI-based therapy. She first took Imatinib for four years; however, her molecular response was suboptimal according to the European Leukemia Net guidelines (2009) and this treatment was not well tolerated. Imatinib was therefore replaced with Dasatinib and following TKI replacement, she was in major molecular response for approximately 10 months before expressing her wish to become pregnant. As this scenario had not been reported in the literature and did not meet the criteria 
for TKI therapy discontinuation as defined by Mahon et al., it was decided to temporarily discontinue Dasatinib and substitute this treatment with the immunomodulator interferon during the two pregnancies [6]. The TKI therapy with Dasatinib was then resumed.

It is crucial to carefully plan conception and pregnancy to assure the best outcomes for both the mother and the child. The therapeutic plan that we presented here limits the occurrence of congenital abnormalities previously observed in children born from TKI-treated mothers. It is recommended to interrupt the TKI treatment before conception whenever possible and this decision should be made based on the molecular response to treatment and in agreement with the patient and her partner.

Currently, most of the data reported for pregnancies occurring while under TKI treatment mainly describe cases of CML patients who were taking first generation TKIs, including Imatinib. Very little clinical and biological data are available for patients treated with second generation TKIs. Our observation therefore provides further information regarding the effects of second generation TKIs on pregnancy, in particular Dasatinib. Clearly, for all CML patients of childbearing age, the question of procreation and the choice of appropriate contraception should be carefully discussed when selecting a TKI treatment: This is crucial in order to allow the patient to plan for a family in the best and most supported conditions. The initial choice of TKI treatment should also be taken into account: The best treatment aimed at reaching a fast therapeutic response will play a key role in the patient's possibility to plan for a family.

\section{Conclusions}

This case report highlights the possibility today for CML patients to envisage becoming pregnant without jeopardizing their disease status and outcome or exposing the fetus to TKI associated teratogen risks.

\section{Acknowledgments}

The authors would like to acknowledge Aurélie Boulachin.

The authors declare having received grants, consulting fees, financial support for travel to meetings, fees for participating in review activities and for providing writing assistance and payments for lectures from Bristol-Myers-Squibb, IPSOGEN, NOVARTIS, ROCHE, SANDOZ and SHIRE.

\section{References}

[1] M.L. Hensley, J.M. Ford, Imatinib treatment: Specific issues related to safety, fertility and pregnancy, Semin. Hematol. 40 (2 Suppl 2) (2003) 21-25.

[2] P. Ault, H. Kantarjian, S. O'Brien, S. Faderl, M. Beran, M.B. Rios, et al., Pregnancy among patients with chronic myeloid leukemia treated with imatinib, J. Clin. Oncol. 24 (7) (2006) 1204-1208.

[3] L. Garderet, R. Santacruz, V. Barbu, J. van den Akker, B. Carbonne, N.C. Gorin, Two successful pregnancies in a chronic myeloid leukemia patient treated with imatinib, Haematologica 92 (1) (2007) 9-10.

[4] J. Cortes, S. O'Brien, P. Ault, G. Borthakur, E. Jabbour, B. Bradley-Garelik, et al., Pregnancy outcomes among patients with chronic myeloid leukemia treated with dasatinib, in: 50th ASH Annual Meeting, San Francisco, California, USA December 6-9, 2008.

[5] M. Conchon, S.S. Sanabani, I. Bendit, F.M. Santos, M. Serpa, P.E. Dorliac-Llacer: Two successful pregnancies in a woman with chronic myeloid leukemia exposed to nilotinib during the first trimester of her second pregnancy: Case study, J. Hematol. Oncol. 2 (2009) 42-45.

[6] E. Chelysheva, A. Turkina, T. Kolosheinova, G. Gusarova, M. Sokolova, M. Vakhrusheva, et al., Pregnancy outcomes and treatment regimens in patients with chronic myeloid leukemia, in: 17th Congress of EHA, Amsterdam, June 14-17, 2012.

[7] F.X. Mahon, D. Réa, J. Guilhot, F. Guilhot, F. Huguet, F. Nicolini, et al., Discontinuation of imatinib in patients with chronic myeloid leukaemia who have maintained complete molecular remission for at least 2 years: The prospective, multicentre Stop Imatinib (STIM) trial, Lancet Oncol 11 (11) (2010) 1029-1035. 